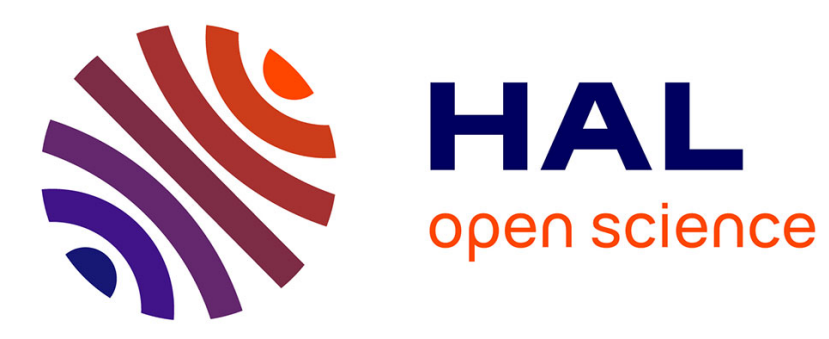

\title{
Simplex Based Adaptive Parametric Model Order Reduction For Applications In Optimization
}

Mateus Antunes Oliveira Leite, Benoît Delinchant, Jean-Michel Guichon, Joao Vasconcelos

\section{- To cite this version:}

Mateus Antunes Oliveira Leite, Benoît Delinchant, Jean-Michel Guichon, Joao Vasconcelos. Simplex Based Adaptive Parametric Model Order Reduction For Applications In Optimization. Optimization and Inverse Problems in Electromagnetism, Sep 2016, Rome, Italy. 10.1002/jnm.2264 hal-01368512

\section{HAL Id: hal-01368512 https://hal.science/hal-01368512}

Submitted on 20 Apr 2020

HAL is a multi-disciplinary open access archive for the deposit and dissemination of scientific research documents, whether they are published or not. The documents may come from teaching and research institutions in France or abroad, or from public or private research centers.
L'archive ouverte pluridisciplinaire HAL, est destinée au dépôt et à la diffusion de documents scientifiques de niveau recherche, publiés ou non, émanant des établissements d'enseignement et de recherche français ou étrangers, des laboratoires publics ou privés. 


\title{
SIMPLEX BASED ADAPTIVE PARAMETRIC MODEL ORDER REDUCTION FOR APPLICATIONS IN OPTIMIZATION
}

\author{
Mateus A. O. Leite ${ }^{1,2}$, Benoit Delinchant ${ }^{1}$, Jean-Michel Guichon ${ }^{1}$ and João A. Vasconcelos ${ }^{2}$ \\ (1) G2ELAB, Univ. Grenoble Alpes, CNRS, Grenoble INP, G2Elab, F-38000 Grenoble, France \\ E-mail: mateus.leite@g2elab.grenoble-inp.fr, benoit.delinchant@g2elab.grenoble-inp.fr and Jean- \\ Michel.Guichon@g2elab.grenoble-inp.fr \\ (2) Graduate Program in Electrical Engineering - Federal University of Minas Gerais - Av. Antônio Carlos 6627, \\ 31270-901, Belo Horizonte, MG, Brazil. E-mail: maol@ufmg.br and joao@cpdee.ufmg.br
}

\begin{abstract}
A new methodology for optimization using parametric reduced order models is introduced. An adaptive scheme to place the expansion points in the reduction step is combined with a smart parametric space sampling in the optimization step to produce very reliable reduced order models. The methodology is illustrated in a demonstrative electromagnetic problem with appreciable gain in computational time. This technique is of general application and can be used to speed up optimization processes in many areas of engineering.
\end{abstract}

Keywords: moment matching, parametric model order reduction, dynamic systems

\section{Introduction}

In the last decades, very successful numerical modeling techniques have been developed and studied. The Finite Elements Method (FEM) or the Partial Element Equivalent Circuit (PEEC), for example, are capable of building models that have a high degree of precision. It is, however, hard to directly use these models for simulation and optimization. If the required accuracy is very high or if the physical system is too complex, high dimensional models may be produced. It is in this context that Model Order Reduction (MOR) may be applied. Given a High-Fidelity Model (HFM), the objective of this technique is to build a Reduced Order Model (ROM) that approximates the input/output behavior of the HFM but that has a much lower dimension, allowing faster computations [1]. There are well-established methods for this purpose like Balanced Truncation [2], Proper Orthogonal Decomposition [3] and Moment Matching [4]. For the reduction of equivalent circuits obtained by the PEEC method, algorithms like PRIMA [5] and SPRIM [6] have been shown to be particularly efficient.

If MOR techniques were to be used to speed up simulation processes, one would need the ROM to conserve the parametric characteristics of the HFM for the design process [7]. This would allow an optimization to be lunched without the high cost of using the HFM for each objective function evaluation. One approach to perform this task is to build several ROMs for different parameter values and perform model interpolation for parameter values different from the sampled points. However, this approach will become very expensive for a large number of parameters [8].

In this article, we present an adaptive methodology for producing very reliable reduced order models and a smart strategy for sampling the parameter space to minimize the number of model order reductions during optimization processes. Both of these tasks are crucial for an effective optimization methodology based in MOR. One cannot expect to obtain correct interpolated models if the models used as basis for the interpolation are not precise. At the same time, it is 
1 important to keep the number of sampled models as low as possible to reduce the computational 2 burden of the whole optimization process.

3 This article deals with systems like the one in (1): linear systems in descriptor form. In these 4 equations $A$ and $E \in \mathbb{R}^{n \times n}, B \in \mathbb{R}^{n \times p}, C \in \mathbb{R}^{q \times n}, x \in \mathbb{R}^{n}, u \in \mathbb{R}^{p}$ and $y \in \mathbb{R}^{q}$. The number $n$ is the order of the model and $p$ and $q$ are, respectively, the number of inputs and outputs of the system. The vector $x$ is the state vector of the dynamical system and $u$ and $y$ are its input and output vectors, respectively.

This set of equations is the natural form of writing many physical systems like circuits. At the same time, the developed methodology is very general and may be adapted to different fields of applications. This generality comes from the fact that no hypothesis is made about the structures of the matrices of the state space. Any system that can be written in this form can be 12 treated.

$$
\left\{\begin{aligned}
E \frac{d x}{d t} & =A x+B u \\
y & =C x
\end{aligned}\right.
$$

This article is divided in four main sections. The first one introduces the main subject. Section 2 presents a review of reduced order models based in Krylov Subspaces and introduces the technique to place the expansion points adaptively. The smart sampling of the parametric space for parametric model order reduction is described in Section 3. Section 4 ends the article with a conclusion.

\section{Model Order Reduction}

Projection based model order reduction is a framework that reduces the model by a projection of the state variables into a lower dimension subspace [8]. Each method will differ in the way that this subspace is chosen. For practical reasons, the subspaces are represented as matrices whose columns span the subspace.

If one projects a system represented by (1) into the subspace spanned by $V$ and orthogonal to the span of $W$, the result is given by (2). In many applications, $W$ is chosen as being equal to $V$. For different purposes, the $\mathrm{W}$ matrix can be chosen to guarantee a stable or passive reduced order model [9].

$$
\left\{\begin{array}{c}
W^{T} E V \frac{d x_{r}}{d t}=W^{T} A V x_{r}+W^{T} B u \\
y_{r}=C V x_{r}
\end{array}\right.
$$

To keep notation as simple as possible, one can introduce some definitions as in (3).

$$
\begin{array}{cc}
W^{T} E V=E_{r} & W^{T} A V=A_{r} \\
W^{T} B=B_{r} & C V=C_{r}
\end{array}
$$

This leads to a new state space model in descriptor form (4) that can be used instead of the HFM to frequency domain and time domain simulations, if it is accurate enough.

$$
\left\{\begin{array}{c}
E_{r} \frac{d x_{r}}{d t}=A_{r} x_{r}+B_{r} u \\
y_{r}=C_{r} x_{r}
\end{array}\right.
$$


1 The following subsections presents how to choose the matrix $V$ to assure very good accuracy

2 between the high-fidelity model and the reduced one.

\subsection{Moment Matching}

If one applies the Laplace transformation to the system of equations in (1), it is possible to isolate a matrix $(\mathrm{H})$ that relates inputs and outputs as written in (5). For the special case of a single input and single output (SISO), this matrix reduces itself to the well-known scalar transfer function.

$$
H=C(E s-A)^{-1} B
$$

8 For reasons that will become evident in the next steps, one can write (5) into a slightly different

9 form, shown in (6). In this Equation, $\mathrm{s}_{0}$ is an arbitrary complex number.

$$
H=-C\left(A-s_{0} E-\left(s-s_{0}\right) E\right)^{-1} B
$$

Using the easily proven identity (7) in (6), it is possible to expand this equation to a more useful

11 form, written in (8).

$$
(\mathbb{I}-M)^{-1}=\sum_{i=0}^{\infty} M^{i}
$$

$$
H=-\sum_{i=0}^{i=\infty} C\left[\left(A-s_{0} E\right)^{-1} E\right]^{i}\left(A-s_{0} E\right)^{-1} B\left(s-s_{0}\right)^{i}
$$

For simplicity, it is possible to define the $\mathrm{i}$-th moment of the function at the point $\mathrm{s}_{0}$ as done in (9).

$$
M_{i}\left(s_{0}\right)=-C\left[\left(A-s_{0} E\right)^{-1} E\right]^{i}\left(A-s_{0} E\right)^{-1} B
$$

This definition allows the writing of the transfer function of the original system in terms of its moments centered at $\mathrm{s}_{0}$. This can be seen in (10).

$$
H=\sum_{i=0}^{i=\infty} M_{i}\left(s_{0}\right)\left(s-s_{0}\right)^{i}
$$

A moment matching technique aims at finding a matrix $V$ that produce reduced order models whose moments are the same as those of the HFM for given expansion points $\left(\mathrm{s}_{0}\right)$ up to a given order. Equation (11) contains the condition for this to be true [10]. In this equation, $\mathcal{K}$ represents the krylov subspace up to the order i. To build such a matrix $V$, the well-known Arnoldi process may be used [11].

$$
\operatorname{span}\{V\} \supset \mathcal{K}\left\{\left(A-s_{0} E\right)^{-1} E,\left(A-s_{0} E\right)^{-1} B, i\right\}
$$

It must be noted that it is possible to match moments around many different expansion points. It is only necessary that the above condition be satisfied for each one of the expansion points individually. This will be largely used in the next section to find a set of expansion points that produce very accurate reduced order models. 
2 During the optimization process, it will be necessary to compute the output for a set of systems 3 having different parameters. Each one of these systems have distinct frequency responses. 4 Consequently, one may have an unreliable frequency response if the placement of the expansion points is not chosen individually for each system.

6 In this section, we provide an adaptive and very reliable heuristic method for the automatic placement of the expansion points. The objective of the method is to find points that provide a reliable frequency response and, at the same time, keeps a low order for the reduced order model. These goals are achieved by doing successive repartitions in the frequency domain and assuring that the frequency response is within an accepted error at each iteration. This idea is analogous to the one used in [12], but in another context.

As seen in the last subsection, the reduced order model obtained by moment matching will have the same moments as those of the high-fidelity model. The 0 -th moment for a given expansion point, is the frequency response of the system for the frequency $\mathrm{s}_{0}$. This can be directly verified by plugging 0 in the value of $i$ in (9). This means that the frequency response of both reduced and high fidelity model are the same at that point. This is the main idea behind the following heuristic, shown as a pseudo algorithm and detailed in the following paragraphs.

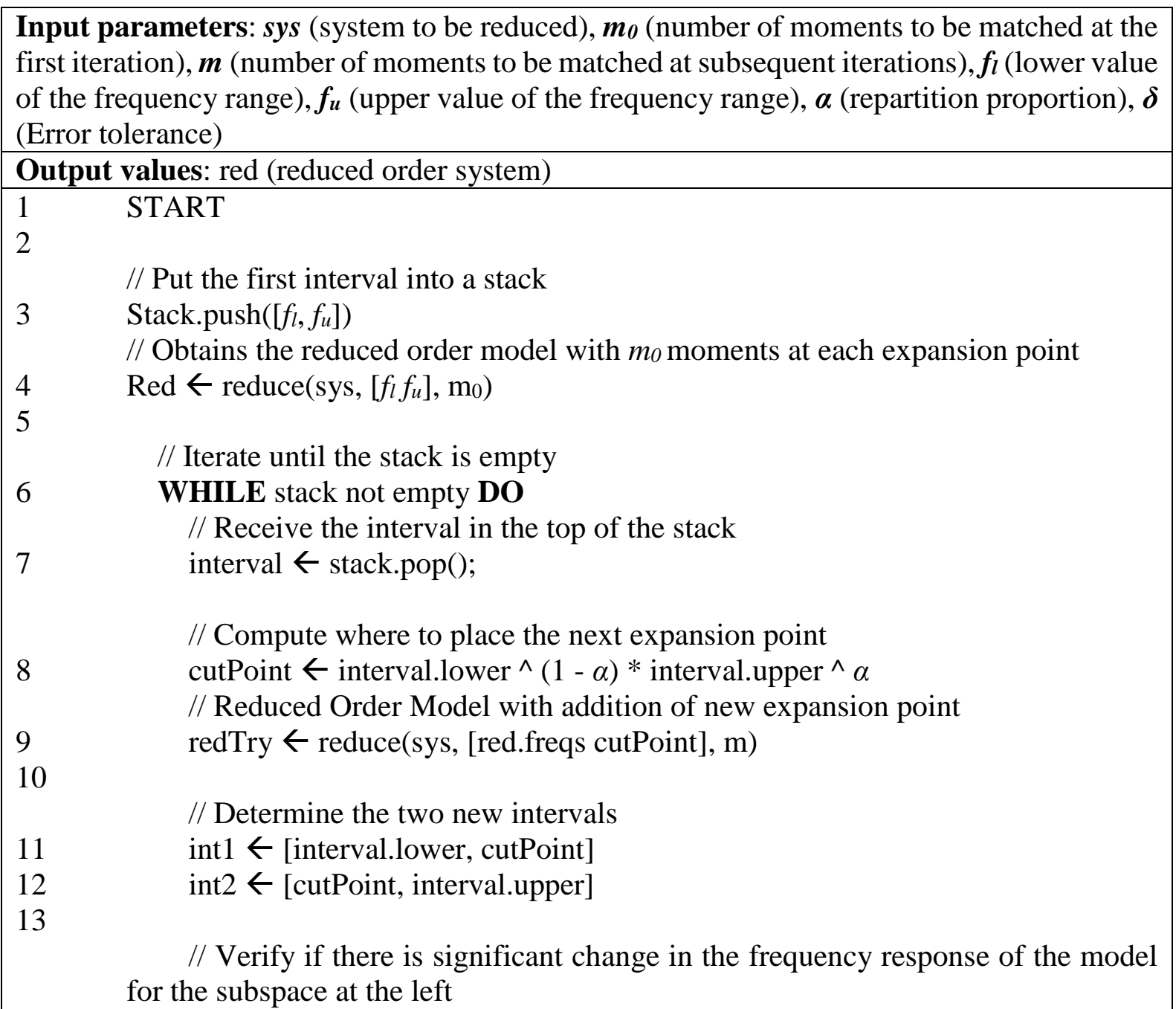




\begin{tabular}{|cc|}
14 & IF $\max (\mid$ simulation(redTry) - simulation $($ red $) \mid>\delta$ ) in int 1 THEN \\
15 & Stack.push(int $)$ \\
16 & Keep $\leftarrow$ true \\
17 & END IF \\
18 & // Verify if there is significant change in the frequency response of the model \\
& for the subspace at the right \\
19 & IF max $(\mid$ simulation(redTry) - simulation(red $) \mid>\delta$ ) in int2 THEN \\
20 & Stac.push(int2) \\
21 & Keep $\leftarrow$ true \\
22 & END IF \\
23 & // If there is significant change, keep the current Reduced Order Model \\
& IF Keep is true THEN \\
24 & red $\leftarrow$ redTry \\
26 & END IF \\
27 & END WHILE \\
28 & END $\quad$ \\
\hline
\end{tabular}

2 The user must supply lower and upper bounds for the frequency range of interest and an 3 acceptable error tolerance. These bounds are used to build the first frequency interval. Each 4 extreme receives one expansion point with $\mathrm{m}_{0}$ moments matched $\left(\mathrm{m}_{0}=2\right.$ by default). This will produce a first reduced order model of very low order but that is exact for the chosen point and matches the first derivative of the full model. This model and the whole interval delimited by the lower and upper bounds supplied by the user are placed in a stack (FIFO data structure). At each iteration on interval of the stack is analyzed and process is repeated until the structure is empty.

At each iteration, the interval in the top of the stack is split in two new intervals. A new expansion point is chosen using Equation (12), where $f_{e}, f_{l}$ and $f_{u}$ are, respectively, the frequencies of the expansion points, lower and upper frequencies values of the interval.

$$
f_{e}=f_{l}^{1-\alpha} f_{u}^{\alpha}
$$

This equation reduces to a geometric mean if $\alpha$ is one half, the default choice. A new reduced order model and its frequency response are obtained. It must be pointed out that the projection subspaces obtained for previous iterations are not recomputed. The algorithm only proceeds with the computation of the vectors associated to the new point. After the addition of this new expansion point with $m$ moments matched ( $m=1$ by default), two new frequency intervals will have been delimited, one between the lower bound and the expansion point and a second one from the expansion point to the upper bound. The order of the moments to be matched are somehow arbitrary. If $m$ is chosen to be one, this means that the model will be exact for the selected expansion point and no derivatives are matched. There is indeed a trade-off between accuracy and computational cost.

The difference of the frequency response is computed for each one of these intervals. If any of the values is bigger than the accepted precision, this interval is added to the stack. Otherwise, it is discarded and one considers that the algorithm has converged for that region. It is Important to note that if the interval is discarded, the expansion point is not kept in the final reduced order 
model. If there is no change, this means that the information added by the new point was already present in the model. Therefore, keeping it would increase the order of the model without a correspondent increase in accuracy. This guarantees that the order of the ROM is as small as possible.

This method was applied to two different systems of different domains: a cantilever beam obtained by finite elements methods [13] and a loop antenna obtained by the PEEC method [14]. The beam receives the force at the extreme of the beam and outputs the total displacement. It consists of a set of 1200 equations. The loop antenna is excited by a unitary current source and outputs the impedance of the system for different frequencies. The total number of equations for this system is 1035 . For both systems, the accepted error tolerance is of $0.1 \mathrm{~dB}$ and the frequency range where $1 \mathrm{~Hz}-1 \mathrm{kHz}$ and $1 \mathrm{Ghz}-20 \mathrm{GHz}$, respectively. Figure 1 presents these results.

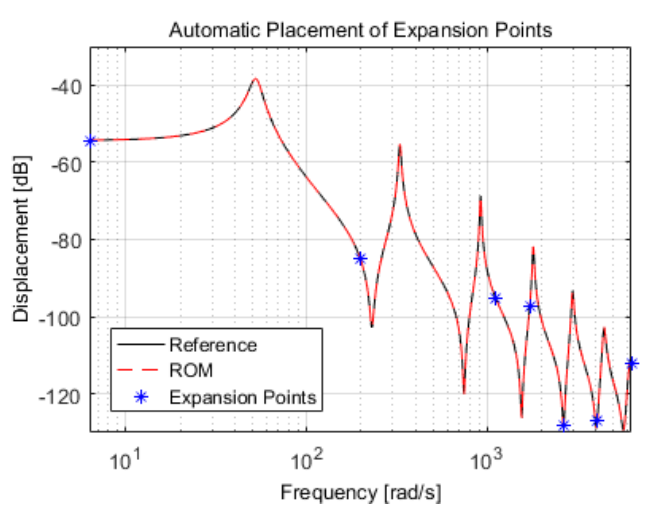

(a)

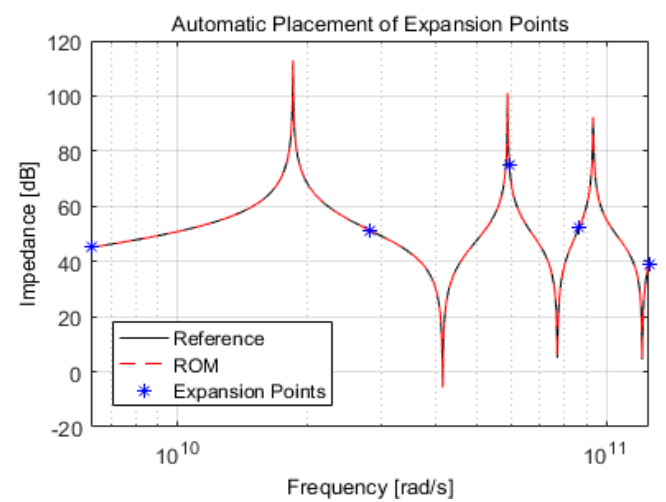

(b)

Figure 1 (a) Comparison of the ROM ant the HFM for the cantilever beam. (b) Comparison of the ROM ant the HFM for loop antenna.

It must be noted that all the computations are done only using successive reduced order models. The High-Fidelity Model is never used for a comparison. The simulation of the ROM can be done in a very efficient way using the Hessenberg algorithm in [15]. Thus, this process is very fast. The orders of the obtained system are 14 and 18, respectively, compared to the originals of 1200 and 1035, respectively. Even though the final reduced order models are much smaller than the high-fidelity ones, the adaptive algorithm has successfully chosen points that leads to very good approximations, as can be seen by the comparison between the reference and the obtained Reduced Order Models.

\section{Parametric Model Order Reduction for Optimization}

The process of model order reduction is capable of producing a very good approximation of one given model with a certain parameter set. However, for the optimization process it is necessary to evaluate the behavior of these models for different values of the parameters at each iteration. Reducing a new model for each of these points may be very time consuming. To overcome this difficulty, one may use techniques of parametric model order reduction [8]. Many methods like [16] and [17] need a kind of parameter space sampling as a starting point to produce interpolated models. For the optimization process, equally sampling the whole parameter space is not useful. During the execution of the algorithm only regions with 
1 promising values of the objective function will be consulted. This particularity can be used to 2 guide the sampling strategy to be used. The next subsection presents a way of doing this.

\subsection{Choosing the sampling points with a simplex based strategy}

In polynomial interpolation, one provides measures for a set of independent variables and intermediate values are obtained by an interpolating scheme. For the case of model interpolation, the process is analogous. The user must provide a set of known models for different parameter sets and an interpolating strategy is used to produce intermediate models.

One may choose the sampling points in very different manners. Naive strategies like building a hypercube in the parameter space will only work for very low dimensional parameter spaces due to the curse of dimensionality. To overcome this problem, strategies based on spare grids may be applied [18]. However, it is stills inefficient if an optimization process is the target application. One does not need to sample to whole parameter space, only the regions that are requested by the optimization algorithm.

To do this, we propose a new strategy based on a simplex, the generalization of a triangle to an arbitrary number of dimensions. When the optimization process starts, one must provide a starting point. A simplex having this point as its center is build using a process like the one presented in [19]. For the sake of completeness, we briefly describe this procedure. In this discussion, $n$ is the dimension of the space and, consequently, the simplex is composed of $n+$ 1 vectors. One starts by building a symmetric matrix $M \in R^{n \times n}$ as shown in (13).

$$
M_{i j}=\left\{\begin{array}{c}
1 \quad \text { if } \quad i=j \\
-\frac{1}{n} \quad \text { if } \quad i \neq j
\end{array}\right.
$$

The columns of the upper triangular Cholesky factor of this matrix contains the first $n$ vectors that compose the simplex. The last one can be computed by taking the negative of the sum of the first $n$ vectors. A simplex that is not centered at zero can be obtained by a simple translation of the obtained points. In a similar fashion, its points can be rotated to obtain different simplexes. The ideal size of the simplex is a problem that is left as an open issue. For the current discussion, physical knowledge of the problem can be used to infer an appropriate size.

At each one of the simplex vertices, a High-Fidelity Model is reduced using the adaptive strategy presented in Section $2 \mathrm{~b}$. As long as the optimization algorithm keeps analyzing points inside this simplex, interpolated models are used to evaluate the objective function. When the algorithm steps out of the simplex, the farthest point from the current solution is reflected. The Figure 2 shows an illustration of this procedure and identifies the interpolation zone and a possible new reflected point. 


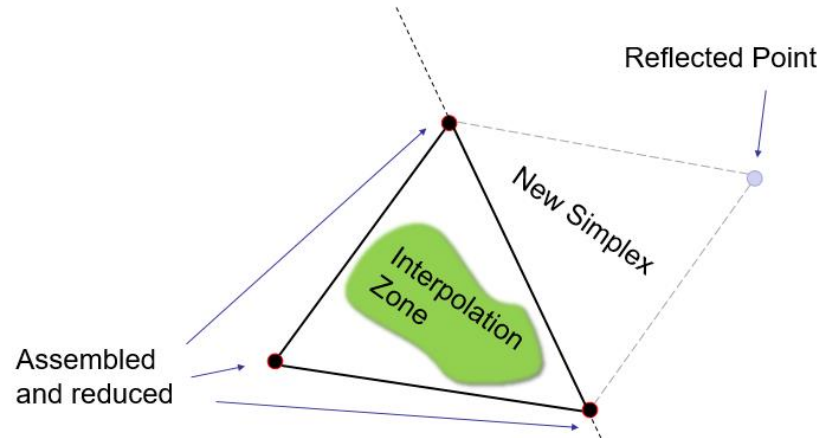

3 To successfully implementing this method, one needs a way to detect if a point is contained on 4 the simplex. This can be easily done by using the convex hull definition of a simplex. If all the 5 coefficients of this linear combination in (14) are positive, the point is inside the simplex, and 6 outside otherwise. In this equation, the vectors $v_{i}$ are the vectors from the origin to the i-th 7 vertex of the simplex and $\mathrm{p}$ is the point to be analyzed.

$$
p=\sum_{i=1}^{n+1} \alpha_{i} v_{i}
$$

8 Equation (15) imposes the constant that the weights of this linear combination all sum up to 9 one.

$$
\sum_{i=1}^{n+1} \alpha_{i}=1
$$

10 These two equations can be arranged as a linear system to allow direct resolution. Notice that 11 there is a slight abuse of notation in (16) to write the matrix composed of vectors and scalars.

$$
\left[\begin{array}{cccc}
v_{1} & v_{2} & \cdots & v_{n+1} \\
1 & 1 & \cdots & 1
\end{array}\right]\left[\begin{array}{c}
\alpha_{1} \\
\alpha_{2} \\
\vdots \\
\alpha_{n+1}
\end{array}\right]=\left[\begin{array}{l}
p \\
1
\end{array}\right]
$$

12 The simplex will follow the current solution in a way that only very few points are sampled in 13 the parameter space, resulting in a very few reductions processes.

\subsection{Subspace Interpolation}

The main goal of projection based model order reductions is to find a suitable subspace in which the state space vector should be projected in order to reduce the number of unknowns and at the same time produce accurate input/output behavior. It is then natural to use the subspaces obtained for different parameter values in order to produce a reduced order models in a quicker manner [17].

20 To achieve this in a systematic way, one can use a very successful technique that is the 21 interpolation of these subspaces in a tangent space to the Grassmann manifold. 
1 For the case of only one variables, one can interpret subspace interpolation as being a process that transforms the principal vectors of one subspace into those of a second one by means of rotation. This idea was used to deduce suitable formulas for this particular case in [20] and [21]. Later, this idea was generalized by [17] to deal with an arbitrary number of dimensions. For the optimization process, the generalized process described in [17] and resumed in [8] is used.

\subsection{Illustration of the method}

For illustrating the efficacy of the developed methodology, an electromagnetic optimization problem is solved using this process. It consists of maximizing the impedance, in a certain frequency range, between a set of conductor wires inside a cage. Figure 3 shows the difference of the landscape for the full system and the reduced and interpolated one. It is possible to see that both have the same tendency. The simplex used for this computation has a side of $50 \mathrm{~mm}$ and the grid step is $2 \mathrm{~mm}$. The maximum error obtained is of $23.7 \%$ at $(0.2794,0.4980)$, a point in the boundary of the domain. The mean absolute error is $0.05 \%$. One can conclude that even though in some place the error is appreciable, in average, the interpolated model allows very accurate predictions.

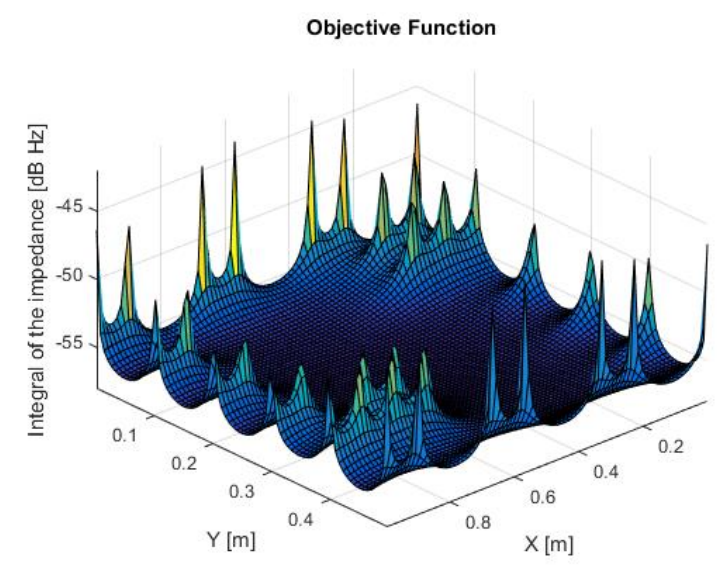

(a)

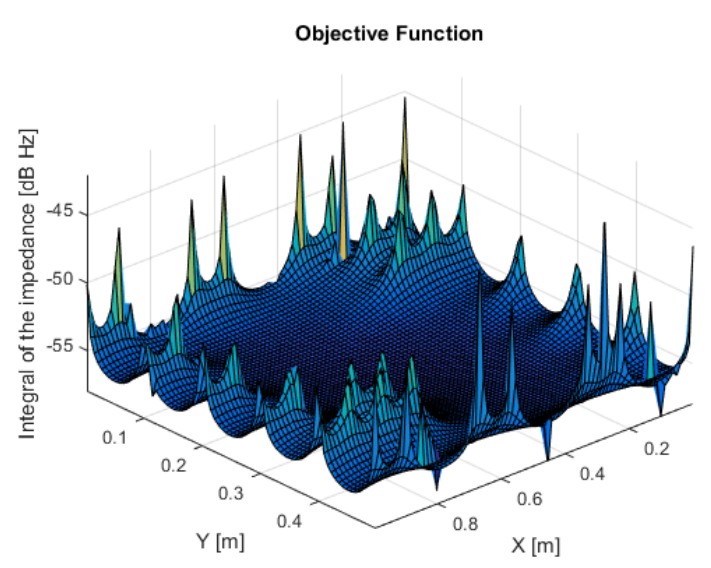

(b)

Figure 3 (a) Objective Function surface for the high-fidelity model. (b) Objective function surface for the interpolated model.

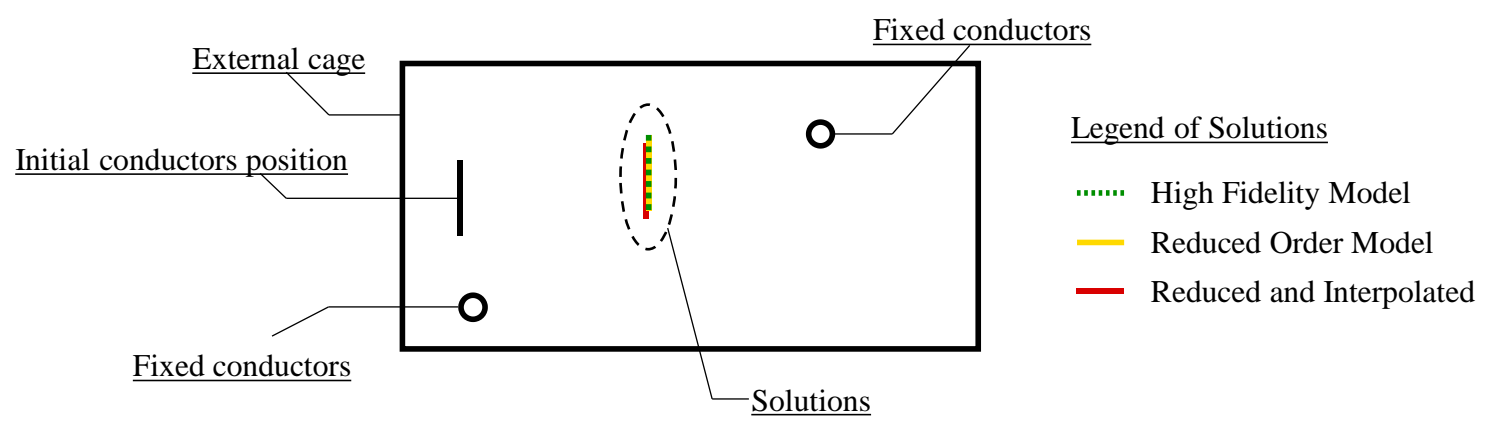

Figure 4 Result for the optimization problem for the high-fidelity model, reduced model and reduced and interpolated model.

Figure 4 shows the result of the didactic optimization problem for the High-Fidelity Model, the Reduced Order one and for the Reduced and Interpolated Model. It is possible to conclude that all the processes have almost found the same solution, but with very different computational 
1 times. The distance of the simplex nodes to its geometric center were chosen to be $90 \mathrm{~mm}$, 2 which results in an area of approximately $2.1 \%$ of the area of the domain.

3 Table 1 shows in details the results of all the optimization processes. Table 2 contains the 4 number of times that the different optimization processes used the high-fidelity model, number 5 of reductions and interpolations. Figure 5 shows the progression of the optimization algorithm 6 and the corresponding simplexes that were constructed to perform the interpolations.

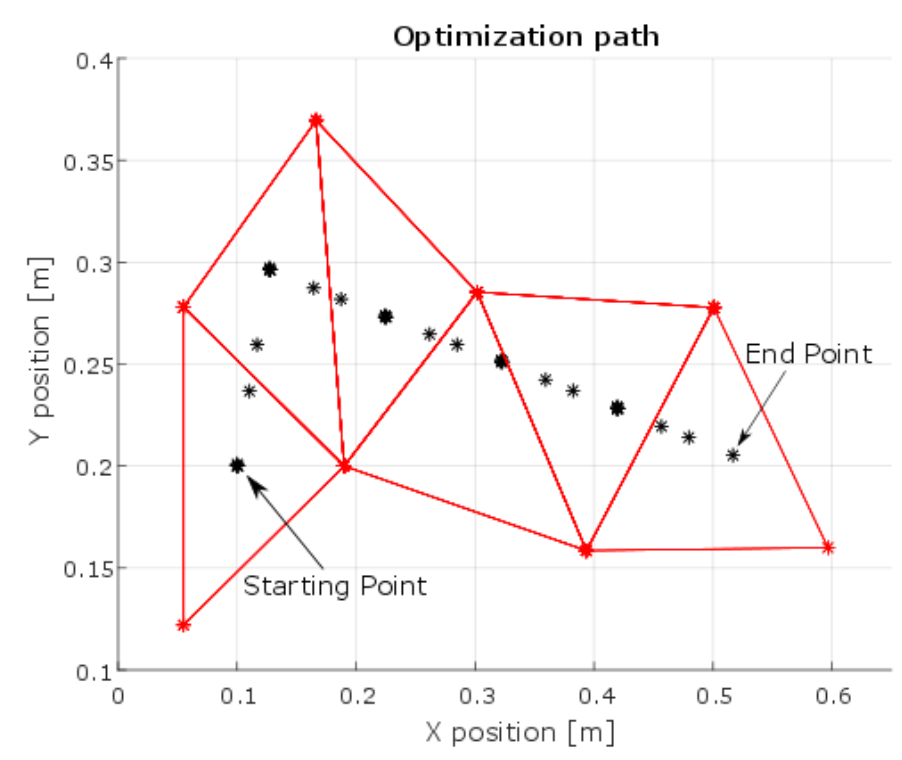

Figure 5 - Example of the simplexes following the optimization path

\section{Conclusion}

A complete methodology for optimization using parametric model order reduction to speed up objective function evaluations was presented. It has the advantage of lowering the computational cost of the process while keeping high accuracy. A very efficient heuristic to adapt automatically the placement of the expansion points was developed. It was combined with a smart parameter space sampling strategy to compose a very solid methodology for optimization large-scale dynamical systems. Further work is needed in order to determine an automatic way of choosing the size of the simplex.

The presented technique is applicable to any system that can be written in a linear state space formulation, being applicable to many different domains of engineering.

\section{References}

[1] A. C. Antoulas, Approximation of Large-scale Dynamical Systems, Society for Industrial and Applied Mathematics, 2005.

[2] B. Moore, "Principal component analysis in linear systems: Controllability, observability, and model reduction," Automatic Control, IEEE Transactions on, vol. 26, pp. 17-32, Feb 1981. 
[3] G. a. H. P. a. L. J. L. Berkooz, "The proper orthogonal decomposition in the analysis of turbulent flows," Annual review of fluid mechanics, vol. 25, no. 1, pp. 539--575, 1993.

[4] R. W. Freund, "Krylov-subspace methods for reduced-order modeling in circuit simulation," Journal of Computational and Applied Mathematics , vol. 123, pp. 395421, 2000.

[5] A. Odabasioglu, M. Celik and L. T. Pileggi, "PRIMA: passive reduced-order interconnect macromodeling algorithm," IEEE Transactions on Computer-Aided Design of Integrated Circuits and Systems, vol. 17, pp. 645-654, Aug 1998.

[6] R. W. Freund, "SPRIM: structure-preserving reduced-order interconnect macromodeling," in Computer Aided Design, 2004. ICCAD-2004. IEEE/ACM International Conference on, 2004.

[7] A. Alla, M. Hinze, O. Lass and S. Ulbrich, "Model order reduction approaches for the optimal design of permanent magnets in electro-magnetic machines," IFACPapersOnLine, vol. 48, pp. 242-247, 2015.

[8] P. Benner, S. Gugercin and K. Willcox, "A Survey of Projection-Based Model Reduction Methods for Parametric Dynamical Systems.," SIAM Review, vol. 57, pp. 483-531, 2015.

[9] B. N. Bond and L. Daniel, "Guaranteed stable projection-based model reduction for indefinite and unstable linear systems," in Computer-Aided Design, 2008. ICCAD 2008. IEEE/ACM International Conference on, 2008.

[10] M. S. Hossain, "Numerical methods for model reduction of time-varying descriptor systems," 2011.

[11] W. E. ARNOLDI, "THE PRINCIPLE OF MINIMIZED ITERATIONS IN THE SOLUTION OF THE MATRIX EIGENVALUE PROBLEM," Quarterly of Applied Mathematics, vol. 9, pp. 17-29, 1951.

[12] L. H. de Figueiredo, "Adaptive sampling of parametric curves," Graphics Gems V, vol. 5, pp. 173-178, 1995.

[13] H. Panzer, J. Hubele, R. Eid and B. Lohmann, "Generating a parametric finite element model of a 3D cantilever Timoshenko beam using MATLAB," Tech. reports on aut. control, Inst. Aut. Control, TU M^"u\}nchen, 2009.

[14] F. Freschi, G. Gruosso and M. Repetto, "Unstructured PEEC formulation by dual discretization," Microwave and Wireless Components Letters, IEEE, vol. 16, pp. 531533, Oct 2006.

[15] A. Laub, "Efficient multivariable frequency response computations," in 19th IEEE Conference on Decision and Control including the Symposium on Adaptive Processes, 1980.

[16] H. Panzer, J. Mohring, R. Eid and B. Lohmann, "Parametric model order reduction by matrix interpolation," at-Automatisierungstechnik Methoden und Anwendungen der Steuerungs-, Regelungs-und Informationstechnik, vol. 58, pp. 475-484, 2010. 
[17] D. Amsallem and C. Farhat, "Interpolation method for adapting reduced-order models and application to aeroelasticity," AIAA journal, vol. 46, pp. 1803-1813, 2008.

[18] S. A. Smolyak, "Quadrature and interpolation formulas for tensor products of certain classes of functions," in Dokl. Akad. Nauk SSSR, 1963.

[19] A. Conn, K. Scheinberg and L. Vicente, Introduction to Derivative-Free Optimization, Society for Industrial and Applied Mathematics, 2009.

[20] T. Lieu and M. Lesoinne, "Parameter adaptation of reduced order models for threedimensional flutter analysis," AIAA Paper, vol. 888, p. 2004, 2004.

[21] T. Lieu and C. Farhat, "Adaptation of aeroelastic reduced-order models and application to an F-16 configuration," AIAA journal, vol. 45, pp. 1244-1257, 2007. 
Table 1 Results of the optimization process

\begin{tabular}{|c|c|c|c|c|}
\hline & $X[\mathrm{~m}]$ & $Y[\mathrm{~m}]$ & Objective Function $[\mathrm{dB} \mathrm{Hz}]$ & Time $[\mathrm{s}]$ \\
\hline HFM & 0.4230 & 0.2431 & -55.5542 & 624 \\
\hline ROM only & 0.4232 & 0.2431 & -55.5543 & 163 \\
\hline ROM and Interpolation & 0,4195 & 0,2282 & -55.5611 & 65 \\
\hline
\end{tabular}

3

4

Table 2 Number of reductions, interpolations and calls to the high-fidelity model

\begin{tabular}{|c|c|c|c|}
\hline & Model Reductions & Model Interpolations & HFM Calls \\
\hline HFM & 0 & 0 & 36 \\
\hline ROM only & 36 & 0 & 0 \\
\hline ROM and Interpolation & 8 & 28 & 0 \\
\hline
\end{tabular}

